\title{
Behind the scenes of a big hybrid conference
}

\author{
The 2021 Materials Research Society (MRS) Fall Meeting was a hybrid event for the first time, featuring both an \\ in-person meeting in Boston and a virtual meeting held separately. Nature Reviews Materials speaks to Gopal Rao, \\ Chief Editor of Technical Content for MRS and Editor of MRS Bulletin, to find out what pandemic-era conference \\ planning is like.
}

\begin{abstract}
Q The hybrid format of this MRS meeting is unique, with in-person and virtual events held on different weeks, and different line-ups and registrations. Can you tell us what led to the decision to organize separate events rather than having virtual attendees log in live to the Boston event?

The hybrid format was developed partly to enhance the meeting experience for in-person and virtual meeting attendees, and partly owing to logistical reasons. All the special talks and a few regular sessions in Boston were live-streamed to the virtual audience. In addition, we encouraged all presenters in Boston to upload recordings of their talks to our virtual meeting platform that could be viewed asynchronously by the virtual meeting attendees (and in-person attendees as well). All in-person attendees were able to fully participate in the virtual meeting the following week, which would have been difficult for them to do if the two meetings had overlapped.

There was a large focus on in-person networking. We knew that networking was of paramount importance to the in-person attendees' experience after nearly 2 years without in-person interactions. We wanted to reserve ample time in the programme for attendees to interact. This split schedule also allowed logistically for better organization of the two meeting components, which translated to a better meeting experience for in-person and virtual meeting attendees.
\end{abstract}

We knew that networking was of paramount importance to the in-person attendees' experience after nearly 2 years without in-person interactions
Q In hosting a separate virtual event, is MRS aiming to recreate the same feel and experience as an in-person conference, or are you trying to redefine what a virtual experience can be?

We are indeed trying to redefine what a virtual meeting experience can be - by marrying the best aspects of an in-person meeting with the benefits afforded by a virtual meeting. In addition to listening to scientific talks, interactions with other researchers and networking are critical parts of the in-person meeting experience. We tried to recreate this to the extent possible in a virtual environment. The virtual environment has its own advantages of course. Those who cannot attend an in-person meeting for various reasons can participate. Attendees can asynchronously watch talks at their convenience if they are unable to attend the sessions when scheduled - like watching TV shows using DVR. Also, all the recordings will remain available to view on demand through to 15 January 2022. It is likely that future MRS meetings past the pandemic will continue to include a virtual meeting component for those who may not be able to make it to the in-person meeting. Our current experience with the virtual meetings is helping to set the stage for future virtual meetings.

Q In planning this meeting, were there discussions about inclusivity?

A MRS Core Value is to be egalitarian and inclusive. MRS has a long history of efforts in this area, including MRS Women in Materials Science Programs, the Inclusive Graduate Education Network, and the MRS Developing Country programme. Earlier this year, the MRS Board of Directors approved a DEI Aspiration, stating "The Materials Research Society embraces diversity, equity, and inclusion (DEI) by actively engaging our diverse global membership, supporting the careers of underrepresented researchers, and investing in community actions to address bias and end inequities.” To advance this aspiration, MRS

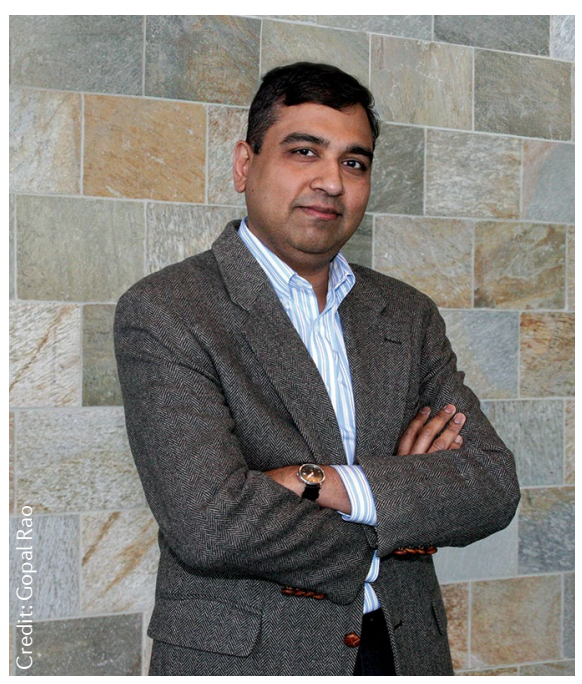

held a Diversifying Materials event in Boston at the Fall Meeting, launching six new special interest groups (SIGs) to give our members from underrepresented minority populations a place to connect, collaborate and advise MRS on ways to create a more inclusive culture.

In terms of the meeting itself, MRS has always strived to make our meetings accessible to researchers in countries or regions who may find it difficult to come to Boston or the USA. The virtual meetings make it possible for a researcher anywhere in the world to be able to attend and participate, as we found in our last two virtual-only meetings. During the virtual portion of the 2021 MRS Fall Meeting, MRS engaged more than 100 underrepresented minority students in the MRS PREM (Partnerships for Research and Education in Materials) Scholars programme, which included professional development programmes and the opportunity to present their research during a virtual poster session. Additionally, the virtual component of the meeting also enables inclusion of researchers who otherwise would be unable to participate in the meeting. 


\section{Q How about sustainability and reducing environmental impact?}

There were indeed discussions on reduced environmental impact of the in-person and virtual components of the hybrid meeting. We had been heading towards more sustainable meetings even before the pandemic, for instance by reducing the printed programmes and abstracts and moving meeting information to an online/app format. Reduced travel of course helps the environment by reducing carbon emissions. But this has to be balanced by the advantages afforded by an in-person meeting. The hybrid format for the MRS Fall Meeting allowed for a more sustainable meeting. It is fitting that sustainability was also the topic under discussion in some of the symposia at the meeting! There were symposia on materials for sustainable electronics, sustainable batteries, sustainable conversion of atmospheric gases into fuels and chemicals, and climate change mitigation technologies. MRS also has a group focused on sustainability efforts that drives programming in meetings and publications.

Q Did you see a change in the type of registrations - for example, geographical location for the virtual meetings in 2020 and spring 2021, compared to in-person meetings pre-pandemic? Have there been differences in registration between the in-person and virtual portions of this fall's hybrid meeting?

We did observe some variations in registration types for the last two virtual MRS meetings, with more attendees from typically underrepresented countries, presumably because no travel was needed. For this hybrid meeting, the proportion of in-person and virtual attendees was very close to 50:50. There were fewer non-US in-person attendees in Boston than usual, as expected for the present situation. We will be looking at the registrations and demographics closely for the MRS Fall Meeting in the coming weeks.

Q. Have you received feedback from the materials science community about what they like and dislike about virtual meetings? Has the community expressed any clear preferences? MRS has been regularly surveying our meeting attendees and the broader materials community to gain insights on their thoughts on virtual meetings overall, on MRS meetings more specifically, and how their views have changed since the pandemic began. We have been refining our planning based on the feedback provided. After more than 1.5 years of the pandemic and numerous online conferences, meetings, classes and discussions, indications are that the materials science community is ready for face-to-face interactions and in-person meetings. Scientists thrive on in-person discussions and interactions, the raison detre for scientific meetings and conferences. In addition to hearing talks and new research, other interactions at scientific conferences are crucial, to discuss ideas and set up collaborations. These are missed in virtual meetings.

Virtual meetings, while necessary in a pandemic, only work to an extent. Anecdotally, we are hearing a lot about 'Zoom fatigue'. The message is that people do not like the absence of face-to-face interactions in virtual meetings. We also hear that Q\&A and discussions following scientific talks are not as robust in a virtual environment. Another aspect missing from virtual meetings is the 'running into' colleagues that happens by chance at in-person meetings. The absence of in-person networking is a major factor. With that said, we believe that virtual meetings are here to stay and need to continue to evolve as a complement to the in-person experience.

What does the future look like for MRS meetings? Do you think MRS will continue to offer a virtual or hybrid option after travel restrictions and risks lift, or is the plan to go back to in-person-only events?

The plan is to largely go back to in-person meetings, but we will continue to have virtual components for upcoming meetings post-pandemic. Even without travel restrictions and health concerns, some potential attendees may not be able to travel to an event. The virtual option allows them to participate, while at the same time expanding the breadth of scientific content presented at our meetings. We are looking forward to the 2022 MRS Spring Meeting scheduled to be held in Honolulu, Hawaii, in May. This meeting has already attracted the largest number of symposium proposals submitted to an MRS meeting and also one of the highest number of abstracts submitted to an MRS meeting. We anticipate very strong international, especially Pac-Rim, participation.

Virtual events are not just confined to MRS Spring and Fall Meetings. They also remain an option for events in-between larger meetings and for workshops. The MRS Webinars series has significantly expanded over the past 2 years and has been very well received by the community. We have held several workshops in collaboration with other scientific societies and have an inaugural MRS Communications/Nature Communications Workshop on Advances in Materials and Manufacturing on 25-26 January 2022. These expanded virtual offerings have increased our engagement of the materials community between the MRS Spring and Fall meetings.

Q What were the main challenges in planning this meeting? What would you do differently next time?

Given the timing for the planning for this meeting, the major challenge was the uncertainty in what this meeting would look like, whether it would be a hybrid meeting or a fully virtual meeting. The planning for MRS meetings starts a couple of years before each event. Planning for this meeting thus happened during the pandemic, including core planning during the second wave of the pandemic this year as well as amidst uncertainties associated with the Delta variant of the SARS-CoV-2 virus. We did not know whether international attendees would be able to travel to Boston, what protocols would be in place for the health and safety of our attendees and staff, and whether mandates would allow our venues to host the meeting. Hence, we made the abstract submission and meeting registration process very flexible. This did mean that we were operating with many unknown variables, making the overall planning process difficult. For future meetings, we are hoping to set the right balance between flexibility and certainties in the processes. We will continue to ensure that the safety of our meeting attendees and MRS staff remains a high priority.

Q What is your technology wish list for an ideal virtual conference? What is still missing that would vastly improve the virtual experience?

We evaluated many virtual platforms at the onset of the pandemic, and each has its own pros and cons. What we discovered is that there are only a few that could handle the complexity of the MRS meeting structure reasonably well, and our vendor partner has been great in working with us on making improvements from each virtual meeting to the next. New advancements in virtual and hybrid meeting technologies are being developed every day, and we continue to monitor those for solutions that will provide the easiest and most streamlined way for our members to share their research and network. Quality 'impromptu' networking - the human connection - is something that is lacking in the virtual environment. However, whether or not a technology solution can be developed to replicate that remains to be seen.

Interviewed by Ariane Vartanian

https://doi.org/10.1038/s41578-022-00414-3 